\title{
Atenção básica de saúde e a assistência em Hanseníase em serviços de saúde de um município do Estado de São Paulo
}

\author{
The basic health and assistance to Hansen's Disease in health care services of a municipality of São Paulo State
}

La atención básica a la salud y la asistencia a la Lepra en servicios de salud de un municipio del Estado de São Paulo

\author{
Adriana Jimenez Pereira', Lúcia Maria Frazão Helene', Elisete Silva Pedrazini", \\ Cleide Lavieri Martins ${ }^{\text {III }}$ Carmen Silva de Campos Almeida Vieira ${ }^{\text {IV }}$ \\ 'Universidade de São Paulo. Escola de Enfermagem. Departamento de Enfermagem em Saúde Coletiva. São Paulo, SP \\ "Universidade Federal de São Carlos. Departamento de Enfermagem, São Carlos, SP \\ I'Universidade de São Paulo. Faculdade de Saúde Pública, Departamento de Prática. São Paulo, SP \\ "Universidade de Taubaté.Departamento de Enfermagem. Taubaté, SP
}

Submissão: $28 / 08 / 2008$

Aprovação: 10/10/2008

\section{RESUMO}

Este é um estudo descritivo desenvolvido em um município do Estado de São Paulo. Objetivo: identificar e caracterizar as ações do Programa de Controle da Hanseníase nos serviços de saúde municipais. Metodologia: entrevistas gravadas com gestor municipal de saúde e profissionais da assistência à hanseníase. Resultados: a política pública municipal em saúde prioriza o desenvolvimento da atenção básica com ênfase na saúde pública. As ações são realizadas por profissionais capacitados e experientes em hanseníase. Verificou-se a não realização da busca ativa dos casos, necessária para o real conhecimento da situação epidemiológica, e das ações de educação em saúde, importante para a redução do estigma e aproximação do sujeito à nova situação de vida e enfrentamento de limitações.

Descritores: Hanseníase; Serviços de saúde; Atenção básica à saúde

\section{ABSTRACT}

This descriptive study was carried out in a municipality of Sao Paulo State. The objective was to identify and to characterize the Leprosy Control Program in primary care in the city of research. Methodology: the interviews were conducted with professionals responsable for care in Hansen's Disease and with manager of public health policies. Results: the public health policies prioritize the development of primary care and emphasis on the public health. The control actions are held by trained and experient professionals. It emphasizes absence of active search for cases which undermines the real epidemiological situation, and the important health education to the reduction of stigma and bring the subject of adjustments necessary in new situation of life and overcome limitations.

Descriptors: Leprosy; Health services; Primary health care.

\section{Resumen}

Este estudio descriptivo es una investigación que analizó la situación de la atención de la Lepra en un municipio del Estado de Sao Paulo. Objetivo: identificar y caracterizar las acciones del Programa de Control de Lepra de los servicios de salud de ese municipio. Metodología: se entrevistaron a los profesionales encargados de la atención de lepra y al director municipal de políticas de salud. Resultados: las políticas públicas municipales de salud priorizaron el desarrollo de la atención básica, con énfasis en la salud pública tradicional. Las acciones de control de lepra son realizadas por trabajadores capacitados y con significativa experiencia profesional. Se resalta la ausencia de busca activa de los casos, necesaria para un conocimiento real de la situación epidemiológica y la importancia de educación en salud, para reducir el estigma y aproximar el sujeto a las adaptaciones necesarias en la nueva situación de vida para afrontar las limitaciones.

Descriptores: Lepra; Servicios de salud; Atención primária de salud. 


\section{INTRODUÇÃO}

As últimas décadas foram marcadas por intensas transformações no sistema de saúde brasileiro, relacionadas com as mudanças ocorridas no âmbito político-institucional e, simultaneamente, ao processo de redemocratização iniciado nos anos 80. Neste contexto, foi constituído o Sistema Único de Saúde (SUS), regulamentado pela Constituição Federal de 1988 e pelas Leis no 8080/90 e $\mathrm{n}^{\circ}$ 8. 142/90 (Lei Orgânica da Saúde), Que prevê por meio de seus princípios e diretrizes o acesso universal, igualdade no atendimento assistencial e equidade na distribuição dos recursos ${ }^{(1)}$.

Diante das recomendações atuais do SUS, é de competência dos municípios a organização dos serviços da atenção básica, e atribui ao Estado, os papéis de normatização, avaliação e assessoria técnica. Dessa forma, os municípios assumem a responsabilidade como gestores da saúde e à rede básica de saúde cabe a competência de prestar a assistência à população e assim, em específico, aos doentes com hanseníase.

Apesar dos avanços observados no controle da hanseníase, a endemia ainda apresenta coeficientes epidemiológicos alarmantes em alguns Estados brasileiros, situação Que classifica o Brasil entre os noves países (Angola, Brasil, República Central Africana, República Democrática do Congo, Índia, Madagascar, Moçambieue, Nepal e República Unida da Tanzânia) Que não alcançaram, em 2005, a meta de eliminação de hanseníase, menos de I doente para cada 10.000 habitantes ${ }^{(2)}$.

No Brasil, no ano de 2005, a taxa de prevalência da hanseníase diminuiu para 1,48 casos por 10 mil habitantes, o que equivale a 27.313 pessoas em tratamento. O coeficiente de detecção foi de 2,09 por 10 mil habitantes, o que equivale a 38.410 casos novos notificados pelos serviços de saúde ${ }^{(2)}$. Diante dessa realidade, foram estabelecidos estratégias e esforços para Que até o ano de 2010 sejam alcançados coeficientes correspondentes ao previsto na proposta da OMS.

Para compreender a dificuldade de eliminação ${ }^{(1)}$ da hanseníase em nosso país, é necessário considerar a sua grande extensão territorial e principalmente a desigualdade social entre os Estados brasileiros.

Em estudo desenvolvido por Opromolla ${ }^{(3)}$ em 606 municípios do Estado de São Paulo Que possuíam ao menos um caso de hanseníase detectado no período, os autores concluem Que a distribuição da hanseníase no Estado de São Paulo, como em todo o Brasil, ocorre de maneira desigual entre as áreas. Afirmam que os coeficientes de altas taxas médias de detecção nas regiões oeste do Estado de São Paulo, devem estar contribuindo para o aparecimento de novos casos nas regiões Centro- Oeste do país. Também na região Noroeste do Estado observam-se índices elevados desse indicador.

O coeficiente de prevalência do estado de São Paulo, em 2005, era de $1,04 / 10$ mil habitantes, tendo como numerador um total de 4.206 casos em registro ativo ${ }^{(4)}$.

No entanto, o Estado de São Paulo, apresenta cidades com realidades distintas e coeficientes extremos. A cidade de Bauru, local deste estudo, evidencia coeficiente de prevalência considerado como baixo $(0,62 / 10 \mathrm{mil}$ habitantes) e coeficiente de detecção considerado médio $(0,33 / 10 \text { mil habitante })^{(5)}$.

A Proposta de Combate à Hanseníase no Estado de São Paulo, para o período de 2006 a 2010, encontra-se fundamentada na existência de número considerável de municípios Que mantém a cadeia de transmissão da doença, e reforça a necessidade da implementação de estratégias para o diagnóstico precoce e o tratamento de todos os doentes ${ }^{(2)}$. Além da cura bacteriológica dos casos diagnosticados, visa também a realização de ações para prevenir e tratar as incapacidades e os problemas sociais prevalentes nessas regiões $^{(5)}$.

Diante das considerações realizadas, o objeto do presente estudo consiste na situação da assistência à hanseníase, a partir das concepções dos profissionais e do gestor das políticas de saúde municipais, no âmbito dos serviços de saúde do município de Bauru (SP). Os objetivos do trabalho foram: identificar e caracterizar a implementação do Programa de Controle da Hanseníase em serviços de saúde de Bauru tendo como referência o controle da doença e analisar as modificações ocorridas nos serviços de saúde, no período de 2001 a 2006, em relação aos indicadores epidemiológicos municipais.

\section{PROCEDIMENTOS METODOLÓGICOS}

Esta pesquisa está vinculada a um trabalho de investigação maior de autoria de Helene et a $^{(6)}$ denominado: Impacto da organização de serviços de saúde na eliminação da hanseníase em municípios do estado de São Paulo, Que tem como objetivo central evidenciar as formas específicas de produção de serviços de saúde em hanseníase no Estado de São Paulo, de forma a subsidiar a rearticulação da Rede Básica de Saúde, face à Municipalização da saúde e à meta para o controle da hanseníase em nosso país.

O presente trabalho trata-se de um estudo descritivo-exploratório, com base na investigação epidemiológica. Segundo Minayo ${ }^{(7)}$, a pesquisa exploratória compreende as etapas: de escolha do tópico de investigação da pesquisa, de delimitação do problema, definição do objeto e dos objetivos, de construção do marco teórico conceitual, dos instrumentos de coleta dos dados e da exploração do campo. A investigação epidemiológica é um método científico Que possibilita uma aproximação com o objeto e o problema de estudo com a finalidade de obter dados que evidenciam a realidade concreta dos mesmos $^{(8)}$.

O estudo foi realizado no município de Bauru, região oeste do Estado de São Paulo, localizada a $345 \mathrm{~km}$ da capital do Estado, constituída por uma população essencialmente urbana, com 356.680 habitantes, e apresenta taxa de alfabetização em torno de 95\%, segundo Instituto Brasileiro de Geografia e Estatística ${ }^{(9)}$.

A escolha do município se justifica pelo fato deste ser um cenário de transformações históricas e políticas importantes ocorridas com a hanseníase no Estado de São Paulo. A cidade foi sede do antigo asilo-colônia Aimorés, criado para internar doentes com hanseníase de 64 municípios da região, e chegou a abrigar cerca de 1.435 hansenianos. Na época, foi classificado, pelo Departamento de Profilaxia da Lepra, como modelo de colônia asilar ${ }^{(10)}$. Atualmente, constitui-se em um Instituto de Pesquisas em Hanseníase e reconhecido pelo Ministério da Saúde como Centro de Referência Nacional em Hanseníase.

O setor de saúde da cidade estrutura-se, segundo a atenção primária, composto por 24 unidades/núcleos de saúde da rede básica $^{(5)}$.

Para a delimitação do tamanho da amostra, considerou-se a 
Quantidade de unidades básicas de saúde (UBS) existentes no município de Bauru e o tipo de atividades executadas em relação à hanseníase, tendo-se como preocupação manter uma representatividade estatística de 10\% dos serviços existentes. A seleção dos locais de estudo foi realizada por meio de sorteio, totalizando 4 unidades básicas de saúde (UBS) apresentadas a seguir: a UBS I: caracterizada como um serviço de saúde de maior complexidade e de referência para o tratamento dos doentes com hanseníase; as UBS II e UBS III: duas unidades básicas de menor complexidade; e a UBS IV: relacionada com o gerenciamento das políticas públicas da saúde básica, responsável pela administração, planejamento, organização e avaliação das atividades da atenção básica de saúde.

A população de estudo foi composta por todos os profissionais da saúde responsáveis pela assistência à hanseníase nos serviços de saúde selecionados na região estudada. A amostra foi constituída por 10 profissionais responsáveis pela assistência à hanseníase Que concordaram em participar do estudo e Que estavam disponíveis nos serviços de saúde estudados, nos dias agendados para a realização da coleta de dados, inclusive o Secretário Municipal da Saúde, responsável pela gestão das políticas de saúde do município, a fim de analisarmos a conjuntura local e compreender a dimensão política em Que os serviços de saúde estão inseridos.

O estudo foi aprovado pelo Comitê de Ética da Escola de Enfermagem da Universidade de São Paulo (Processo no5 1 4/2005/ CEP-EEUSP) por se tratar de uma investigação no âmbito da saúde Que envolveu a participação de seres humanos como sujeitos da pesquisa, de acordo com o Conselho Nacional de Saúde em sua resolução $n^{\circ} .196$ de 10 de outubro de 1996. A permissão da Secretaria Municipal de Saúde de Bauru para a realização do estudo nos serviços de saúde selecionados também foi consentida. Após os sujeitos da pesquisa serem informados sobre a investigação, garantindo-se o anonimato, foi solicitado o consentimento livre e esclarecido.

A coleta de dados, realizada durante o primeiro semestre de 2007, ocorreu por meio de 3 instrumentos de pesquisa constituídos de Questões abertas e fechadas, direcionados para: os profissionais da saúde (Instrumento I), a interlocutora do Programa de Controle da Hanseníase da DIR 10 (Instrumento II) e o gestor municipal da saúde (Instrumento III). Os instrumentos foram aplicados por meio de entrevistas semi-estruturadas realizadas no local de trabalho dos sujeitos. As entrevistas foram gravadas, em seguida, transcritas e analisadas.

Numa segunda etapa da pesquisa, por meio de um outro instrumento (Instrumento IV), foram registrados e analisados os indicadores epidemiológicos da hanseníase. Neste estudo, as fontes dos indicadores analisados foram as informações existentes na Divisão Técnica de Hanseníase do Centro de Vigilância Epidemiológica Prof. "Alexandre Vranjac" (CVE) da Secretaria de Estado da Saúde de São Paulo que dispõe de bancos de dados de hanseníase de todo o Estado, por Diretoria Regional de Saúde (DIR) e por município.

Dentre os indicadores utilizados para avaliação anual do controle da hanseníase foram pesquisados, no período de 2001 a 2006, os seguintes coeficientes: de prevalência, percentagem de casos paucibacilares $(\mathrm{PB}=\mathrm{I} / \mathrm{T})$, percentagem de casos multibacilares $(\mathrm{MB}=$ $\mathrm{V} / \mathrm{D})$, coeficiente de detecção de casos novos menores de 15 anos e acima de 15 anos de idade, percentagem de casos com deformidade, coeficiente de abandono e percentagem de contatos intrafamiliares examinados.

\section{RESULTADOS E DISCUSSÃO}

\section{Os indicadores epidemiológicos}

A seguir, procederemos à exposição da análise dos dados epidemiológicos da hanseníase em uma série histórica dos últimos 5 anos, referente ao período de 2001 a 2006, tendo como enfoque as ações realizadas de acordo com as diretrizes do Programa de Controle da Hanseníase. A Tabela I apresenta os coeficientes epidemiológicos em hanseníase de 2001 a 2006.

Os dados epidemiológicos do município de Bauru retratam Que, no período analisado, o coeficiente de prevalência dos casos de hanseníase apresentou-se maior no ano de 2003 (0,84 casos / 10 mil habitantes), totalizando 28 casos de hanseníase em tratamento. Os casos multibacilares representavam $83,34 \%$, e os paucibacilares 16,66\%. O coeficiente de detecção, neste mesmo ano, foi também o maior do período estudado, compreendendo 27 casos novos, 26 destes tratam-se de casos novos acima de 15 anos ( 1,04 casos/ 10 mil habitantes), e apenas 01 caso $(0,12$ casos/ 10 mil habitantes) possuía idade entre 0 e 15 anos de idade.

O coeficiente de detecção evidencia forte Queda entre o ano de 2003 a 2006, apresentando em 2006 apenas 06 casos detectados (0,22 casos/ 10 mil habitantes). A redução desse indicador deve ser vista com cautela, pois essa diminuição pode ser efeito da substituição

Tabela 1. Coeficientes de hanseníase, no período de 2001 a 2006, do município estudado. Bauru/São Paulo, 2007.

\begin{tabular}{|c|c|c|c|c|c|c|c|c|c|c|c|c|}
\hline \multirow{2}{*}{ Coeficientes } & \multicolumn{2}{|c|}{2001} & \multicolumn{2}{|c|}{2002} & \multicolumn{2}{|c|}{2003} & \multicolumn{2}{|c|}{2004} & \multicolumn{2}{|c|}{2005} & \multicolumn{2}{|c|}{2006} \\
\hline & $\mathrm{n}$ & $\%$ & $\mathrm{n}$ & $\%$ & $\mathrm{n}$ & $\%$ & $\mathrm{n}$ & $\%$ & $\mathrm{n}$ & $\%$ & $\mathrm{n}$ & $\%$ \\
\hline Detecção de casos novos(0 a 15 anos) & - & 0 & - & 0 & 1 & 0,1 & 1 & 0,1 & - & 0 & - & 0 \\
\hline Detecção de casos novos (> 15 anos) & 8 & 0,3 & 14 & 0,5 & 26 & 1,0 & 15 & 0,5 & 16 & 0,5 & 6 & 0,2 \\
\hline Prevalência & 9 & 0,2 & 16 & 0,5 & 28 & 0,8 & 17 & 0,5 & 16 & 0,5 & 14 & 0,4 \\
\hline Percentagem casos com deformidade & - & 0 & - & 0 & 6 & 20,6 & 3 & 18,7 & 3 & 18,7 & I & 11,1 \\
\hline Percentagem casos multibacilares & - & 0 & - & 0 & 5 & 83,3 & 2 & 66,6 & 3 & 100,0 & 1 & 100,0 \\
\hline Percentagem casos paucibacilares & - & 0 & - & 0 & 1 & 16,61 & 1 & 33,3 & - & 0 & - & 0 \\
\hline Percentagem casos recidivas MB e PB & - & 0 & - & 0 & - & 0 & - & 0 & - & 0 & - & 0 \\
\hline Coeficiente de Abandono & - & 0 & 2 & 12,5 & 4 & 14,2 & 1 & 5,6 & 4 & 25,0 & 1 & 11,2 \\
\hline Percentagem contatos intrafam. & & & & & & & & & & & & \\
\hline Examinados & & 52,3 & & 85,0 & & 96,5 & & 50,0 & & 86,6 & & 90,0 \\
\hline
\end{tabular}


da detecção ativa pela passiva de casos ou mesmo de diagnóstico equivocado.

A porcentagem de casos com deformidade, em 2003, foi 20,64\%, reduzindo-se até 2006 a 11 , 1 I\% dos casos. Segundo Gil Suárez e Lombardi $^{(1 \mathrm{I})}$, o diagnóstico de casos com incapacidades físicas e deformidades indica detecção tardia e, portanto, a presença de casos não detectados numa determinada área. Ressalta-se ainda Que a ocorrência de incapacidade depende diretamente da Qualidade da avaliação do grau de incapacidade física e da Qualidade da informação gerada a partir desta variável.

A porcentagem dos comunicantes examinados apresentou-se alta também no ano de 2003 (96,55\%), passando por elevada Queda em 2004 e estabelecendo-se em 90\% em 2006. Noordeen ${ }^{(12)}$ aponta para o elevado risco de hanseníase apresentado por contatos intradomiciliares, ressaltando assim a importância da prática do exame dos comunicantes para o controle da doença.

\section{Os profissionais e as ações desenvolvidas}

Os 10 profissionais de saúde estudados estavam distribuídos da seguinte forma segundo a função: 2 enfermeiras Que ocupavam a função de gerentes das UBS I e II; I enfermeira Que era responsável pelo Programa de Controle da Hanseníase; 1 médico hansenologista; 2 auxiliares de enfermagem; 1 assistente social, gerente da unidade e I psicóloga da UBS I; I enfermeira interlocutora do Programa de Controle de Hanseníase da UBS IV; e o secretário municipal da saúde que possui a formação de médico veterinário.

Dos profissionais entrevistados, 7 (70\%) possuíam capacitação na área da hanseníase, por meio de especializações e de cursos oferecidos pela regional no Instituto Lauro de Souza Lima.

A capacitação dos profissionais Que realizam assistência em hanseníase constitui um dos resultados esperados com o desenvolvimento do Plano Nacional de Eliminação da Hanseníase, estabelecido pelo Ministério da Saúde, a fim de sustentar a eliminação da hanseníase eneuanto problema de saúde pública nos municípios ${ }^{(2)}$.

Segundo Souza ${ }^{(12)}$, Quanto mais capacitados forem os trabalhadores de saúde, melhor será a assistência às necessidades da população. Desta forma reforçam Que os processos educativos são fundamentais nos serviços de saúde, porém tais processos, Que se dão por meio de capacitação, treinamento ou cursos de longa duração, são pontuais e não abordam criticamente os problemas encontrados na prática profissional, com dificuldades em chegar à essência das Questões para melhor compreender os determinantes fenômenos. A problematização dos processos de trabalho não é vista como instrumento de aprendizagem, o não entendimento das raízes históricas e sociais da prática cotidiana de trabalho faz com Que os processos educativos sejam limitados, não permitindo transformações no serviço.

As equipes de saúde responsáveis pela atenção à hanseníase, segundo os profissionais entrevistados dos locais estudados são compostas da seguinte forma: na UBS I (7 profissionais): I enfermeira, I médico hansenologista, I oftalmologista, I psicóloga, I assistente social e 2 auxiliares de enfermagem; na UBS II (3 profissionais): I enfermeira, I clínico geral, e I pediatra; na UBS III (7 profissionais): I enfermeira, I clínico geral e 5 auxiliares de enfermagem responsáveis pela realização dos curativos.

No presente estudo, as equipes multiprofissionais responsáveis pela assistência à hanseníase variam em sua composição segundo a complexidade do serviço de saúde estudado, bem como as ações desenvolvidas por estes serviços para o controle da hanseníase.

Quanto às ações realizadas nas UBS, verificamos Que os serviços de saúde de menores complexidades (UBS II e UBS III) realizam atividades de acolhimento/suspeição diagnóstica, educação em saúde e vacinação dos contatos. Na UBS III, em caso de suspeita, ocorre, primeiramente, a avaliação do médico clínico geral, e se confirmada a suspeita encaminha-se para UBS I ou Instituto Lauro de Souza Lima.

Na UBS II, em caso de suspeita, a primeira avaliação é realizada pela enfermeira juntamente com o médico, na Qual são feitos o exame físico e o teste de sensibilidade. Após ser notificado o caso suspeito de hanseníase, a pessoa é encaminhada para a UBS I ou Instituto Lauro de Souza Lima.

Para Opromolla ${ }^{(13)}$, as atribuições do nível elementar, serviço básico de saúde Que presta assistência a uma comunidade de até 2000 habitantes, para o controle da hanseníase também deve estar incluída a coleta de material de lesão cutânea e envio da lâmina à unidade de apoio, para exames baciloscópicos; administração de medicamentos conforme es@uema prescrito pelo médico; e aplicação de técnicas simples para prevenção de incapacidades físicas.

Observamos Que a UBS I, considerada referência municipal para a assistência aos doentes de hanseníase, centraliza as ações de tratamento e o acompanhamento dos doentes com hanseníase em Bauru e, realiza as atividades de acolhimento/suspeição diagnóstica, diagnóstico, tratamento, exame dermatoneurológico e vacinação dos contatos, além da notificação dos casos. Segundo Opromolla ${ }^{(14)}$, este serviço é entendido como serviço de nível primário em relação ao controle da hanseníase, atendendo população de 2000 a 20.000 habitantes, e considerado responsável também pelas ações de supervisão e apoio aos demais serviços de saúde.

Os usuários com a suspeita de hanseníase chegam a UBS I encaminhados pelas unidades básicas. Inicialmente, ocorre a avaliação pelo médico dermatologista/hansenologista do serviço que faz a análise de exames complementares e, se necessário, realiza a baciloscopia e a biópsia para a confirmação do diagnóstico. Em seguida, após a notificação do diagnóstico, inicia-se o tratamento com a prescrição da polieuimioterapia, a avaliação do grau de incapacidade, e são realizadas as orientações ao doente na pós consulta pela auxiliar de enfermagem responsável pelo Programa de Hanseníase. Para todos os casos novos, são realizadas pela enfermeira as ações de consulta de enfermagem e/ou visita domiciliária, de acordo com a disponibilidade das poucas salas existentes no serviço, e do tempo devido à grande Quantidade de usuários para serem atendidos no dia, de acordo com o retorno mensal.

As ações de avaliação psicóloga dos doentes ocorrem nos casos em Que o doente verbaliza a necessidade ou Quando o profissional Que o atende evidencia a necessidade imediata. A avaliação oftalmológica e de fisioterapia são realizadas no início e no final do tratamento, e para a avaliação odontológica, os doentes são encaminhados para os outros serviços da cidade que prestam essa assistência, como as clínicas odontológicas das universidades de Bauru (Universidade de São Paulo, Universidade Paulista, Universidade Sagrado Coração).

Foi possível verificar Que a educação em saúde, não incluída nas atividades executadas pela UBS I, é um importante instrumento de controle da hanseníase, Que visa: eliminar falsos conceitos relativos 
à contagiosidade da doença, à incurabilidade e à necessidade de regime de isolamento para o seu tratamento; bem como, orientar Quanto à importância do exame periódico dos contatos, à conveniência do tratamento precoce e à possibilidade de prevenção de incapacidades; estimular a assiduidade do doente, proporcionando atenção individualizada; incentivar a apresentação voluntária de doentes e contatos ${ }^{(14)}$.

As ações educativas, como práticas social e pedagógica, devem ser inerentes a todas as atividades de controle de hanseníase, devem ser desenvolvidas pela equipe de saúde e dirigidas aos usuários, doentes, familiares, instituições e grupos da comunidade. A prática educativa deve permitir a construção e reconstrução de saberes entre a equipe de saúde, usuários do serviço e grupos comunitários, por meio de um processo de ensino aprendizagem participativo, dialógico e problematizador ${ }^{(15)}$.

Evidenciou-se Que a ação de vigilância à saúde de busca ativa dos casos de hanseníase não estava sendo realizada por nenhum dos serviços de saúde estudados. Segundo Mercaroni( ${ }^{(16)}$, a ausência de busca ativa de casos novos pode traduzir um silêncio epidemiológico e retratar uma nova realidade epidemiológica preocupante.

Lastoria e Putinatti ${ }^{(17)}$ afirmam Que a busca ativa periódica em alguns municípios da DIR VIII e da DIR XI, propiciou o descobrimento de casos novos paucibacilares, multibacilares e de comunicantes intradomiciliares, em hanseníase, muitas vezes considerados silenciosos pelos municípios.

Para a descoberta de casos em hanseníase, de acordo com as normas do Centro de Vigilância Epidemiológica devem ser seguidas as ações de: detecção passiva, Que consiste na demanda espontânea da população e nos encaminhamentos feitos por outras unidades; e da detecção ativa Que compreende a busca sistemática de doentes pela equipe da unidade de saúde ${ }^{(5)}$.

Muitas unidades básicas de saúde encaminham os casos suspeitos em hanseníase diretamente para o Instituto Lauro de Souza Lima (ILSL), pois é considerado o centro de referência mais conhecido historicamente pelo cuidado à hanseníase na região. Este fato acarreta uma grande lista de espera para o atendimento no Instituto retardando o atendimento ao novo caso suspeito. A UBS I tem o serviço organizado de forma a priorizar o agendamento dos usuários para a consulta e, tem implantado o tratamento de forma centralizado, exceto na realização de curativos. Esta atividade é realizada nas unidades básicas ou no próprio ILSL devido à falta de estrutura física Que o serviço de referência municipal possui para executar tal assistência.

Segundo a Resolução CFM no 1.780, de 1 I/1 I/2005, Art $1^{\circ}$ parágrafo $3^{\circ}$, os serviços e instituições médico-assistenciais, públicos e privados, devem proporcionar condições para o exercício profissional, disponibilizando exames, medicamentos e outros procedimentos necessários ao diagnóstico e tratamento da hanseníase. Dessa forma, a realização dos curativos aos doentes de hanseníase é de responsabilidade do serviço de saúde Que efetua o tratamento ao doente. Além disso, as normas do Ministério da Saúde preconizam Que o atendimento e tratamento dos pacientes portadores de

\begin{tabular}{|c|c|c|c|}
\hline & UBS II & UBS III & UBS I \\
\hline Área Física & $\begin{array}{l}\text { Considerada Inadequada. Há um } \\
\text { número mínimo de salas para } \\
\text { realizar as atividades, o Que aglutina } \\
\text { diversas atividades numa mesma } \\
\text { sala. }\end{array}$ & $\begin{array}{l}\text { Considerada Inadequada. } \\
\text { As salas são pequenas, Quentes e } \\
\text { pouco iluminadas. }\end{array}$ & $\begin{array}{l}\text { Considerada Inadequada. } \\
\text { Existem poucas salas para } \\
\text { atend imento, e não há sala para } \\
\text { realização de curativos. }\end{array}$ \\
\hline $\begin{array}{l}\text { Auxílio/Apoio } \\
\text { aos doentes } \\
\text { de } \\
\text { hanseníase }\end{array}$ & Não soube informar. & $\begin{array}{l}\text { Cesta básica é oferecida, após } \\
\text { avaliação social, pelo Centro de } \\
\text { Referência e Apoio Social (CRA). }\end{array}$ & $\begin{array}{l}\text { Vale transporte é garantido; cesta } \\
\text { básica destinada advém de outros } \\
\text { Programas; e calçados são } \\
\text { realizados no ILSL. }\end{array}$ \\
\hline $\begin{array}{l}\text { Recursos } \\
\text { Financeiros }\end{array}$ & Falta material de curativos & Falta material de curativos & Falta material de curativos \\
\hline Referência & UBS I ou ILSL & UBS I ou ILSL & $\begin{array}{l}\text { ILSL, para a realização de curativos } \\
\text { e a assistência a maiores } \\
\text { complexidades. }\end{array}$ \\
\hline $\begin{array}{l}\text { Mudança no } \\
\text { Atendimento }\end{array}$ & Não alterou & $\begin{array}{l}\text { Avanços: maior atenção prestada ao } \\
\text { doente com hanseníase; aumento } \\
\text { das informações difundidas sobre a } \\
\text { doença pelos profissionais. } \\
\text { Desgastes: não referido. }\end{array}$ & $\begin{array}{l}\text { Avanços: aumento da capacitação } \\
\text { dos profissionais; maior acesso à } \\
\text { assistência psicológica. } \\
\text { Desgastes: Falta de estrutura física } \\
\text { para realização de curativos; } \\
\text { aumento da demanda das doenças } \\
\text { infecto-contagiosas com o mesmo } \\
\text { número de profissionais e no } \\
\text { mesmo espaço }\end{array}$ \\
\hline $\begin{array}{l}\text { Integração } \\
\text { Serviço- } \\
\text { Ensino } \\
\end{array}$ & $\begin{array}{l}\text { Há recente proposta para também } \\
\text { ser um espaço de estágio para os } \\
\text { estudantes. }\end{array}$ & $\begin{array}{l}\text { Campo de estágio para estudantes } \\
\text { do ensino superior em enfermagem }\end{array}$ & $\begin{array}{l}\text { Campo de estágio para estudantes } \\
\text { do ensino superior em enfermagem }\end{array}$ \\
\hline
\end{tabular}

Quadro 1. Serviços de saúde, segundo a estrutura da assistência à hanseníase, de acordo com a percepção dos sujeitos pesQuisados. Bauru, São Paulo, 2007. 
hanseníase, na rede de atenção básica à saúde, sejam realizados o mais próximo possível às suas respectivas residências ${ }^{(18)}$, Questão dificultada em Bauru devido à necessidade do doente se deslocar, periodicamente, ao ILSL para realizar os curativos.

O Quadro I relaciona os serviços de saúde estudados com a estrutura Que possuem frente à assistência à hanseníase tendo como enfoeue a organização dos serviços de saúde referidas pelos profissionais entrevistados.

As áreas físicas de todas as unidades não possibilitam o desenvolvimento das atividades assistenciais com Qualidade. $\mathrm{O}$ número de salas é insuficiente frente ao número de profissionais e da demanda existente nos serviços, situação Que se agrava na UBS II em Que em uma única sala funciona a administração, a farmácia e também é realiza a pós-consulta; em outra sala ao lado, funciona a emergência, os curativos, a esterilização e o expurgo.

O auxílio destinado aos doentes de hanseníase é, fundamentalmente, o vale-transporte. O Programa de Controle da Hanseníase não dispõe de cesta básica como auxílio, assim o doente de hanseníase Que apresenta tal necessidade recebe cesta básica derivadas de outros programas, como, por exemplo, do Programa de Tuberculose, ou então do Centro de Referência de Assistência Social (CRAS) após avaliação social do doente. Os calçados são confeccionados pelo Instituto Lauro de Souza Lima Que possui sapataria especializada para a adaptação necessária.

Segundo os profissionais entrevistados, os recursos financeiros apresentam-se escassos em relação aos materiais para curativos, sendo que nenhuma das unidades recebe da prefeitura coberturas para o tratamento de lesões.

A UBS I possui como referência para necessidades complexas e realização de curativos o ILSL. As unidades UBS I e UBS II encaminham os suspeitos para o serviço de referência municipal, a UBS I, ou para o ILSL. A escolha entre Qual dos dois serviços é prioridade para o encaminhamento não é definida, em decorrência da falta de informações por parte das unidades básicas.

Em relação às mudanças Que ocorreram no atendimento aos doentes nos últimos cinco anos, o serviço de especialidades atribuiu transformações Que permitiram maior acesso ao tratamento psicológico, e aumento do número de profissionais capacitados, o Que possibilita uma assistência de melhor Qualidade. Na UBS III, as mudanças relacionam-se à maior difusão das informações sobre a doença, aprimoramento do conhecimento e assim maior atenção ao doente.

A integração dos serviços com o ensino esteve presente em todas as instituições estudadas. As UBS I e UBS III são espaços de estágios para estudantes do ensino superior de enfermagem de diversas faculdades da cidade de Bauru, como a Universidade de São Paulo (USP), Universidade Paulista (UNIP), e Universidade Sagrado Coração (USC). A UBS II está em fase de planejamento da integração as universidades, e o primeiro estágio na unidade já está programado.

\section{Análise Qualitativa utilizada para apreensão do objeto sob a ótica dos profissionais}

A análise Qualitativa, de acordo com $\operatorname{Bardin}^{(19)}$, se deu referente a Questões abertas do estudo Que Questionavam sobre a percepção dos profissionais entrevistados Quanto à caracterização dos doentes de hanseníase; e considerações específicas sobre as principais Questões relacionadas à doença. Considerando Que a visão do profissional, sua concepção e valores orientam e fundamenta o exercício da prática.

\section{Condições de Vida dos doentes de Hanseníase}

A vida dos doentes de hanseníase, segundo os depoimentos dos pesQuisados, encontra-se relacionada, principalmente, à baixa situação socioeconômica, refletida em condições de moradia precárias, com falta de higiene, em Que famílias numerosas dividem peQuenos espaços, facilitando a relação do contato íntimo e prolongado entre os familiares:

“... é uma população com uma situação sócio-econômica baixa. Eles têm, normalmente, famílias numerosas, ou tiveram há anos atrás, eu acho que ela está mais relacionada com esse contato íntimo e prolongado" (PI).

“...os de hanseníase, em específico, e os de tuberculose também, são uma população extremamente empobrecida, Que não tem higiene, não tem o Que comer, não é Que não tem higiene porQue não Quer é porQue não têm condição. Assim, às vezes eu vou lá e "Tem que tomar banho", só que chega na casa deles e não tem chuveiro, entendeu...tem um buraco Que cai água. Aí eu falo que tem Que tomar remédio depois da refeição, mas ele não tem o Que comer em casa....." (P3).

“... a hanseníase está sempre ligada com baixas condições socioeconômicas, isso é o principal” (P5).

Essa situação de exclusão também está evidenciada na falta de acesso à educação Que os doentes possuem, elemento Que dificulta muitas vezes a adesão ao tratamento, e o próprio entendimento sobre a doença e suas complicações:

“... eu acho Que as características principais são essas, né, tanto a Questão sócio-econômica, como o desconhecimento, exatamente, da patologia e da possibilidade de cura"(P6).

“... são pacientes de um nível de pobreza, sabe, muito pobres, e assim, eles não têm educação, não tiveram acesso à educação, à escola. Então, o nível de entendimento deles é difícil, são pacientes que você tem que conversar com eles e atender de uma forma muito humanizada, muito calma, sem pressa, de uma forma diferenciada, e não como eles são tratados na maioria dos outros lugares" (P2).

A hanseníase está fortemente associada à intensificação das desigualdades sociais. Os doentes de hanseníase pertencem ao estrato marginal da sociedade, reproduzindo-se em situações precárias de trabalho e vida sob a exclusão social ${ }^{(20)}$.

O profissional da saúde ao aproximar-se da realidade social e cultural do doente, possibilita o desenvolvimento de um trabalho conjunto, co-pactuando as propostas do tratamento, as orientações em relação ao autocuidado e a possíveis adaptações necessárias, a fim de minimizar o impacto da doença na vida dos sujeitos.

\section{Condições de Trabalho dos doentes de Hanseníase}

Os doentes de hanseníase, de acordo com os relatos dos 
profissionais entrevistados, são amputados dos atributos de competência e das capacidades de realizarem tarefas Que possuíam no momento de vida anterior à doença.

“...o trabalho é um segundo momento onde ele, por desconhecimento, na maioria das vezes, ou por dificuldade da mudança de seus costumes, de seus comportamentos, dos hábitos, acabam se seqüelando" (PI).

“...o trabalho está junto né, se não tem trabalho, não tem...mas a gente vê, em geral, Que as pessoas mais necessitadas que desenvolvem a doença, isso é uma coisa Que com o passar dos anos persiste" (P7).

Devido à existência de limitações físicas nos doentes, a exclusão do mundo do trabalho, determinante das formas de produção e reprodução social, pode conferir dificuldades de socialização, o Que reforça a freqüente situação de isolamento Que os doentes possuem nas relações interpessoais.

\section{Representação sobre a aparência dos doentes}

Em nossa cultura, os padrões físicos de beleza são geralmente associados aos padrões morais, assim o belo é relacionado com o bom e os padrões classificatórios de feio induzem à idéia do mau. A pigmentação diferenciada na pele devido às ações medicamentosas e as deformidades Que os doentes podem apresentar são representadas como características definidoras da pessoa Que tem hanseníase, gerando sentimentos de piedade e tristeza pelos profissionais.

"É aquele paciente feinho mesmo, judiação, Que dá pena, certo. Porque falta sensibilidade, entendeu, então é aquele paciente bem visual mesmo, é isso que me vem na cabeça... com aQuela carinha achatadinha de leãozinho, judiação, eles ficam, né, se for deixando de fazer a Quimioprofilaxia, eles ficam assim"(P3).

“ Dá pra perceber a pessoa tem hanseníase... aquela característica de pele diferente, de problema em andar, às vezes, um já está de bengalinha para ajudar a se locomover, não porque ele já tem idade, mas assim, você percebe uma diferença, inchaço no rosto, nas pernas, não sei" (P4).

É fundamental Que o profissional da saúde trabalhe com a autoestima do doente de hanseníase, pois a auto-estima prejudicada induz o sujeito a ocultar a doença ${ }^{(21)}$, ou mesmo isolar-se por se encontrar fora dos padrões hegemônicos de perfeição física.

\section{O Estigma}

Segundo Goffman ${ }^{(22)}$, por definição acredita-se que alguém com um estigma não seja completamente humano. Com base nisso, se faz vários tipos de discriminações, através das Quais efetivamente, e muitas vezes sem pensar, reduzimos suas chances de vida.

Os profissionais dos serviços de saúde estudados relataram que o estigma se trata ainda da principal característica responsável pelo preconceito e pelo isolamento social enfrentado pelos doentes de hanseníase. Embora, há percepção de Que houve mudanças nesse aspecto, devido à evolução do tratamento e diminuição das incapacidades e deformidades, atualmente menos freeüentes no sujeito portador da doença.

“...ainda existe o estigma social, mesmo estando no século XXI a gente vê isso muito claramente" (PI).

“A principal característica é o problema do estigma mesmo, ligado a todos aqueles estigmas que foram se acumulando desde a época antes de Cristo. O principal é o estigma mesmo, é o problema social, o preconceito social que ainda existe e diminui, eu acho Que diminuiu um pouco. Alguns pacientes nem sentem, não percebem, porque como a gente, hoje, fala em hanseníase, eles não relacionam com a lepra, então eles convivem bem na sociedade. Mas a maioria deles sente um impacto social ...eu tenho pacientes, por exemplo agora, Que a família não sabe que ela tem hanseníase, porQue tem medo, medo da reação da família sobre a hanseníase, isso é o principal problema da hanseníase" (P8).

A hanseníase impele as pessoas a viverem situações comuns, em Que surgem marcas produzidas ou reforçadas pela sociedade. Assim, a experiência de adoecer, há séculos, vem sendo associada aos estigmas Que são imputados aos seus doentes ${ }^{(23)}$.

\section{Visão do Gestor Municipal da Saúde}

O depoimento do secretário municipal da saúde do município evidenciou que a cidade de Bauru passa por um processo de reestruturação do modelo de atenção à saúde, priorizando a atenção básica e os dispositivos facilitadores para a implementação das ações de promoção e prevenção da saúde, como a vigilância em saúde, a fim de promover o controle das doenças de notificação compulsória:

“hoje, a destinação que nós estamos fazendo é estritamente na atenção básica, nós estamos reforçando a atenção básica, no sentido de invertermos o modelo, Que era antes centrado em urgência-emergência, Que é um absurdo, foi a vida inteira centrado em urgência-emergência. E, hoje, estamos repactuando isso e direcionando os recursos de forma a fortalecer a atenção básica, ou seja, as ações de promoção e prevenção de saúde, estritamente na rede básica de saúde" (PI0).

“A transformação Que nós estamos favorecendo e vamos implementar ainda até o fim dessa administração, nada mais é do Que concretizar a distritalização da saúde, reforço na atenção básica, vigilância ativa e passiva e a mudança do modelo de gestão centrado na atenção básica e não na urgência e emergência. Eu acho que aí, de supetão, virá todo o controle dessas doenças de notificação obrigatória Que são muito básicas, e Que a gente precisa estar resolvendo com excelência, pra Que, realmente, seja um governo de excelência" (PI0).

Em relação à situação dos serviços de saúde na atenção à hanseníase, considera Que não é uma Questão prioritária, uma vez Que os indicadores epidemiológicos evidenciam o alcance da meta de eliminação, e não relaciona nenhum entrave no atendimento aos doentes. Situação que se opõe aos depoimentos dos profissionais Que realizam a assistência à hanseníase diante das limitações estruturais Que existem nos serviços de saúde. 
“Eu acho Que, em termos de hanseníase, Bauru é uma mosca branca... acho que os dados demonstram muita coisa, os nossos indicadores demonstram, o coeficiente de incidência de hanseníase nosso é muito bom, isso é sinal que pelo menos alguma coisa está sendo feita. ... eu acho que hanseníase nós temos, não só pela, talvez, uma condição regional, mas também a presença desses centros de referência ajudam muito na garantia do acesso do suspeito, do usuário, e também a um bom tratamento" (PI0).

Considera-se Que a função do gerente dos serviços de saúde pública diz respeito a organizar e controlar unidades produtoras de cuidados de assistência médica em redes do setor público, produto das políticas de saúde brasileiras na construção do SUS e do modo peculiar como a saúde foi sendo tomada como Questão social e de Estado $^{24}$. Integrando, assim, o planejamento da produção em saúde às Questões da realidade dos serviços públicos de forma a satisfazer as necessidades dos sujeitos no processo saúde-doença.

\section{CONSIDERAÇÕES FINAIS}

Os resultados obtidos evidenciaram Que as ações de controle da hanseníase em unidades de saúde do município de Bauru, em sua maior parte, são realizadas por profissionais com experiência significativa de trabalho. A evidente capacitação desses profissionais torna-se instrumento fundamental para realizar as ações preconizadas pelo Programa de Controle da Hanseníase. Tal capacitação está fortemente relacionada pela presença do Instituto Lauro de Souza Lima na cidade de Bauru, uma vez Que este é grande pólo de conhecimento e pesquisa na área de hanseníase, o que facilita o acesso dos profissionais a cursos, atualizações e treinamentos nessa área.

A equipe multiprofissional responsável pela assistência dos doentes com hanseníase, nos serviços estudados, varia de acordo com o perfil da unidade de saúde devido a sua complexidade.

Destaca-se a importância de executar constantemente as ações de educação em saúde como prática Que otimiza a adesão ao tratamento e a emancipação do sujeito com hanseníase, devido à superação de limitações provocadas pela doença. Soma-se a isso, a necessidade de implementar a busca ativa frente ao controle da hanseníase para a detecção de casos novos, a possibilidade de em diagnóstico precoce e a concretização da situação epidemiológica no município de Bauru.

Os significados sociais e culturais da hanseníase possibilitam aos profissionais maior aproximação com a realidade do sujeito doente a fim de orientar as práticas em saúde no levantamento das reais necessidades de saúde dos usuários e superar as limitações do tratamento físico-biológico, considerando a problemática social agregada à doença.

A política pública em saúde, em Bauru, prioriza o desenvolvimento da atenção básica, com ênfase na saúde pública tradicional. Nesse contexto, a articulação da gestão com os conflitos inerentes à produção de serviços facilita a integralidade das ações e a Qualidade resolutiva da assistência.

Pretende-se com este artigo contribuir para o redimensionamento do atendimento à hanseníase na rede básica de saúde, no município estudado, com especial atenção à concretização do diagnóstico da realidade da hanseníase. Assim como, fomentar a discussão de estratégias para subsidiar as práticas de serviços de saúde com vistas ao controle da doença.

\section{REFERÊNCIAS}

1. Carvalho GI, Santos L. Sistema Único de Saúde:comentários à Lei Orgânica da Saúde (Lei 8.080/90 e Lei 8.142/90). São Paulo: Hucitec; 1995.

2. Ministério da Saúde (BR). Secretaria de Vigilância em Saúde. Departamento de Vigilância Epidemiológica. Programa Nacional de Eliminação da Hanseníase. Plano nacional de eliminação da hanseníase em nível municipal 2006-2010. Brasília: Ministério da Saúde; 2006.

3. Opromolla PA, Dalben I, Cardim M. Análise da distribuição espacial da hanseníase no Estado de São Paulo, 1991-2002. Rev Bras Epidemiol 2005; 8(4): 356-64.

4. São Paulo. Centro de Vigilância Epidemiológica do Estado de São Paulo - Divisão de Hanseníase Coordenação do Sistema de Vigilância Epidemiológica. São Paulo: SESSP; 2006.

5. Secretaria de Estado da Saúde de São Paulo. Plano de ação para o combate à hanseníase no estado de São Paulo para o período de 2003 a 2006. São Paulo: SESSP; 2002.

6. Helene LMF, Martins CL, Pedrazzani ES,Vieira CSCA, Pereira AJ. Impacto da organização de serviços de saúde na eliminação da hanseníase em municípios do estado de São Paulo; 2005 [em desenvolvimento, financiado pelo $\mathrm{CNP}$ ].

7. Minayo MCS. O desfio do conhecimento: pesquisa Qualitativa em saúde. São Paulo: HUCITEC-ABRASCO; 2004.
8. Breilh J, Granda E. Saúde na Sociedade. Imprensa Oficial do Estado S.A. IMESP. São Paulo: 1986

9 Instituto Brasileiro de Geografia e Estatística. Rio de laneiro: IBGE; 2007.

10. Monteiro YN. Hanseníase: história e poder no Estado de São Paulo. Hansen Int 1987; I 2( I): 1-7.

11. Gil Suárez RG, Lombardi C. Leprosy Elimination at Sub-National Level. Leprosy Review 2000; 71:206-I I.

12. Noordeen SK. The epidemiology of Leprosy. In: Hastings SRC, editor. Leprosy. New York: Churchill Livingstone; 1985. p. 1530.

13. Souza AMA, Galvão EA, Santos I, Roschke MA. Processo educativo nos serviços de saúde. In: Santana JP, Castro IL, organizadores. Capacitação e desenvolvimento de recursos humanos de saúde. Brasília: Ministério da Saúde; 1999. p. 215-32.

14. Opromolla DVA. Noções de Hansenologia. Centro de Estudos Dr. Reynaldo Quagliato. Bauru; 2000.

15. Freire P. Pedagogia da esperança, um reencontro com a pedagogia do oprimido. Rio de Janeiro: Paz e Terra; 2002.

16. Mercaroni D. Análise espacial da Endemia hansênica no município de Fernandópolis /SP [tese]. Ribeirão Preto (SP): Escola de Enfermagem, Universidade de São Paulo; 2003. 
Pereira $\mathrm{Al}$, et al.

17. Lastoria JC, Putinatti MSMA. Utilização de busca ativa de hanseníase: relato de uma experiência de abordagem na detecção de casos novos. Hansen Int 2004; 29(1): 6-I I.

18. Ministério da Saúde (BR). Secretaria de Políticas de Saúde. Departamento de Atenção Básica. Legislação sobre o controle da hanseníase no Brasil. Brasília: Ministério da Saúde; 2000.

19. Helene LMF, Salum MJL. A reprodução social da hanseníase: um estudo do perfil de doentes com hanseníase no município de São Paulo. Cad Saúde Pública 2002; 18(1): 101-13.
20. Bardin L. Análise de conteúdo. Lisboa; Edições 70; 2003.

21. Claro LBL. Hanseníase: representações sobre a doença Rio de Janeiro: FIOCRUZ; 1995

22. Goffman E. Estigma: notas sobre manipulação da identidade deteriorada. Rio de Janeiro: LTC; 1988.

23. Oliveira MHP, Romanelli G. Os efeitos da hanseníase em homens e mulheres: um estudo de gênero. Cad Saúde Pública 1998; 14(I): 51-60. 